\title{
Inclusive production of charged hadrons in photon-photon collisions
}

\section{OPAL Collaboration}

\section{G. Abbiendi ${ }^{\mathrm{b}}$, C. Ainsley ${ }^{\mathrm{e}}$, P.F. Åkesson ${ }^{\mathrm{g}}$, G. Alexander ${ }^{\mathrm{u}}$, G. Anagnostou ${ }^{\mathrm{a}}$, K.J. Anderson ${ }^{\mathrm{h}}$,} S. Asai ${ }^{\text {}}$, D. Axen ${ }^{\text {z }}$, I. Bailey ${ }^{\mathrm{y}}$, E. Barberio ${ }^{\mathrm{g}, 1}$, T. Barillari ${ }^{\text {ae }}$, R.J. Barlow ${ }^{\mathrm{o}}$, R.J. Batley ${ }^{\mathrm{e}}$, P. Bechtle ${ }^{\text {x }}$, T. Behnke ${ }^{\mathrm{x}}$, K.W. Bell ${ }^{\text {s }}$, P.J. Bell ${ }^{\mathrm{a}}$, G. Bella ${ }^{\mathrm{u}}$, A. Bellerive ${ }^{\mathrm{f}}$, G. Benelli ${ }^{\mathrm{d}}$, S. Bethke ${ }^{\text {ae }}$, O. Biebel ${ }^{\text {ad }}$, O. Boeriu ${ }^{\mathrm{i}}$, P. Bock ${ }^{\mathrm{j}}$, M. Boutemeur ${ }^{\text {ad }}$, S. Braibant ${ }^{\mathrm{b}}$, R.M. Brown ${ }^{\text {s }}$, H.J. Burckhart ${ }^{\mathrm{g}}$, S. Campana ${ }^{\mathrm{d}}$, P. Capiluppi ${ }^{\mathrm{b}}$, R.K. Carnegie ${ }^{\mathrm{f}}$, A.A. Carter ${ }^{1}$, J.R. Carter ${ }^{\mathrm{e}}$, C.Y. Chang ${ }^{\mathrm{p}}$, D.G. Charlton ${ }^{a}$, C. Ciocca ${ }^{\mathrm{b}}$, A. Csilling ${ }^{\text {ab }}$, M. Cuffiani ${ }^{\mathrm{b}}$, S. Dado ${ }^{\mathrm{t}}$, A. De Roeck ${ }^{\mathrm{g}}$, E.A. De Wolf ${ }^{\mathrm{g}, 2}$, K. Desch ${ }^{\mathrm{x}}$, B. Dienes ${ }^{\text {ac }}$, J. Dubbert ${ }^{\text {ad }}$, E. Duchovni ${ }^{\text {w }}$, G. Duckeck ${ }^{\text {ad }}$, I.P. Duerdoth ${ }^{\circ}$, E. Etzion ${ }^{\text {u }}$, F. Fabbri ${ }^{\text {b}}$, P. Ferrari ${ }^{\mathrm{g}}$, F. Fiedler ${ }^{\text {ad }}$, I. Fleck ${ }^{\mathrm{i}}$, M. Ford ${ }^{\mathrm{o}}$, A. Frey ${ }^{\mathrm{g}}$, P. Gagnon ${ }^{\mathrm{k}}$, J.W. Gary ${ }^{\mathrm{d}}$, C. Geich-Gimbel ${ }^{\text {c }}$, G. Giacomelli ${ }^{\text {b }}$, P. Giacomelli ${ }^{\text {b }}$, M. Giunta ${ }^{\mathrm{d}}$, J. Goldberg ${ }^{\mathrm{t}}$, E. Gross ${ }^{\mathrm{w}}$, J. Grunhaus ${ }^{\text {u}}$, M. Gruwé ${ }^{\text {, }}$, A. Gupta ${ }^{\text {h }}$, C. Hajdu ${ }^{\text {ab }}$, M. Hamann ${ }^{\text {x }}$, G.G. Hanson ${ }^{\text {d }}$, A. Harel ${ }^{\text {t, }}$ M. Hauschild ${ }^{\mathrm{g}}$, C.M. Hawkes ${ }^{\mathrm{a}}$, R. Hawkings ${ }^{\mathrm{g}}$, G. Herten ${ }^{\mathrm{i}}$, R.D. Heuer ${ }^{\mathrm{x}}$, J.C. Hill ${ }^{\mathrm{e}}$, D. Horváth ${ }^{\mathrm{ab}, 3}{ }^{\text {, }}$

P. Igo-Kemenes ${ }^{\mathrm{j}}$, K. Ishii ${ }^{\mathrm{v}}$, H. Jeremie ${ }^{\mathrm{q}}$, P. Jovanovic ${ }^{\mathrm{a}}$, T.R. Junk ${ }^{\mathrm{f}, 4}$, J. Kanzaki ${ }^{\mathrm{v}, 5}$, D. Karlen ${ }^{\mathrm{y}}$,

K. Kawagoe ${ }^{v}$, T. Kawamoto ${ }^{\text {v }}$, R.K. Keeler ${ }^{y}$, R.G. Kellogg ${ }^{\mathrm{p}}$, B.W. Kennedy ${ }^{\mathrm{s}}$, S. Kluth ${ }^{\text {ae }}$, T. Kobayashi ${ }^{\text {v }}$, M. Kobel ${ }^{\text {c,6 }}$, S. Komamiya ${ }^{\text {v }}$, T. Krämer ${ }^{\text {x }}$, A. Krasznahorkay Jr. ${ }^{\text {ac,7 }}$, P. Krieger ${ }^{\text {f,8 }}$, J. von Krogh ${ }^{\mathrm{j}}$, T. Kuhl ${ }^{\mathrm{x}}$, M. Kupper ${ }^{\mathrm{w}}$, G.D. Lafferty ${ }^{\circ}$, H. Landsman ${ }^{\mathrm{t}}$, D. Lanske ${ }^{\mathrm{m}}$, D. Lellouch ${ }^{\mathrm{w}}$,

J. Letts ${ }^{9}$, L. Levinson ${ }^{\text {w }}$, J. Lillich ${ }^{\mathrm{i}}$, S.L. Lloyd ${ }^{1}$, F.K. Loebinger ${ }^{\circ}$, J. Lu ${ }^{\text {z,10 }}$, A. Ludwig ${ }^{\mathrm{c}, 6}$, J. Ludwig ${ }^{\text {i }}$, W. Mader ${ }^{\text {c,6 }}$, S. Marcellini ${ }^{\text {b }}$, A.J. Martin ${ }^{1}$, T. Mashimo ${ }^{\text {v }}$, P. Mättig ${ }^{11}$, J. McKenna ${ }^{\text {z }}$, R.A. McPherson ${ }^{y}$, F. Meijers ${ }^{g}$, W. Menges ${ }^{\mathrm{x}}$, F.S. Merritt ${ }^{\mathrm{h}}$, H. Mes ${ }^{\mathrm{f}, 12}$, N. Meyer ${ }^{\mathrm{x}}$, A. Michelini ${ }^{\mathrm{b}}$, S. Mihara ${ }^{\text {v }}$, G. Mikenberg ${ }^{\text {w }}$, D.J. Miller ${ }^{\text {n }}$,W. Mohr ${ }^{\text {i }}$, T. Mori ${ }^{\mathrm{v}}$, A. Mutter ${ }^{\mathrm{i}}, \mathrm{K}_{\text {. Nagai }}{ }^{1}$, I. Nakamura ${ }^{\mathrm{v}, 13}$, H. Nanjo ${ }^{\mathrm{v}}$, H.A. Neal ${ }^{\text {af }}$, R. Nisius ${ }^{\mathrm{ae}}$, S.W. O'Neale ${ }^{\mathrm{a}, \mathrm{w}}$, A. Oh ${ }^{\mathrm{g}}$, M.J. Oreglia ${ }^{\mathrm{h}}$, S. Orito ${ }^{\mathrm{v}, *}$, C. Pahl ${ }^{\text {ae }}$, G. Pásztor ${ }^{\mathrm{d}, 14}$, J.R. Pater ${ }^{\mathrm{o}}$, J.E. Pilcher ${ }^{\mathrm{h}}$, J. Pinfold ${ }^{\text {aa }}$, D.E. Plane ${ }^{\mathrm{g}, *}$, O. Pooth ${ }^{\mathrm{m}}$, M. Przybycieńn ${ }^{\mathrm{g}, 15}$, A. Quadt ${ }^{\mathrm{ae}}$, K. Rabbertz ${ }^{\mathrm{g}, 16}$, C. Rembser ${ }^{\mathrm{g}}$, P. Renkel ${ }^{\mathrm{w}}$, J.M. Roney ${ }^{\mathrm{y}}$, A.M. Rossi ${ }^{\text {b }}$, Y. Rozen ${ }^{\text {t }}$, K. Runge ${ }^{\text {i }}$, K. Sachs ${ }^{\text {f }}$, T. Saeki ${ }^{\text {v }}$, E.K.G. Sarkisyan ${ }^{\text {g, } 17}$, A.D. Schaile ${ }^{\text {ad }}$, O. Schaile ${ }^{\text {ad }}$, P. Scharff-Hansen ${ }^{\mathrm{g}}$, J. Schieck ${ }^{\text {ae }}$, T. Schörner-Sadenius ${ }^{\mathrm{g}, 18}{ }^{18}$, M. Schröder ${ }^{\mathrm{g}}$, M. Schumacher ${ }^{c}$, R. Seuster ${ }^{\mathrm{m}, 19}$, T.G. Shears ${ }^{\mathrm{g}, 20}$, B.C. Shen ${ }^{\mathrm{d}}$, P. Sherwood ${ }^{\mathrm{n}}$, A. Skuja ${ }^{\mathrm{p}}$, A.M. Smith ${ }^{\mathrm{g}}$, R. Sobie ${ }^{\mathrm{y}}$, S. Söldner-Rembold ${ }^{\mathrm{o}}$, F. Spano ${ }^{\mathrm{h}, 21}$, A. Stahl ${ }^{\mathrm{m}, 22}$, D. Strom ${ }^{\mathrm{r}}$, R. Ströhmer ${ }^{\text {ad }}$, S. Tarem ${ }^{\mathrm{t}}$, M. Tasevsky ${ }^{\mathrm{g}, 23}$, R. Teuscher ${ }^{\mathrm{h}}$, M.A. Thomson ${ }^{\mathrm{e}}$, E. Torrence ${ }^{\mathrm{r}}, \mathrm{D}^{\mathrm{r}}$. Toya ${ }^{\mathrm{v}}$, P. Tran ${ }^{\text {d}}$, I. Trigger ${ }^{\mathrm{g}, 24}$, Z. Trócsányi ac,7, E. Tsur ${ }^{\mathrm{u}}$, M.F. Turner-Watson a ${ }^{\mathrm{a}}$, I. Ueda ${ }^{\mathrm{v}}$, B. Ujvári ac,7,

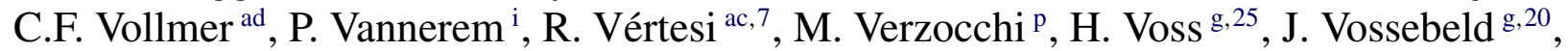
C.P. Ward ${ }^{\text {e}}$, D.R. Ward ${ }^{\mathrm{e}}$, P.M. Watkins ${ }^{\mathrm{a}}$, A.T. Watson ${ }^{\mathrm{a}}$, N.K. Watson ${ }^{\mathrm{a}}$, P.S. Wells ${ }^{\mathrm{g}}$, T. Wengler ${ }^{\mathrm{g}}$, N. Wermes ${ }^{\text {c }}$, G.W. Wilson ${ }^{0,26}$, J.A. Wilson ${ }^{\mathrm{a}}$, G. Wolf ${ }^{\mathrm{w}}$, T.R. Wyatt ${ }^{\mathrm{o}}$, S. Yamashita ${ }^{\mathrm{v}}$, D. Zer-Zion ${ }^{\mathrm{d}}$, L. Zivkovic ${ }^{\mathrm{t}}$

\footnotetext{
a School of Physics and Astronomy, University of Birmingham, Birmingham B15 2TT, UK

${ }^{\mathrm{b}}$ Dipartimento di Fisica dell' Università di Bologna and INFN, I-40126 Bologna, Italy c Physikalisches Institut, Universität Bonn, D-53115 Bonn, Germany
} 


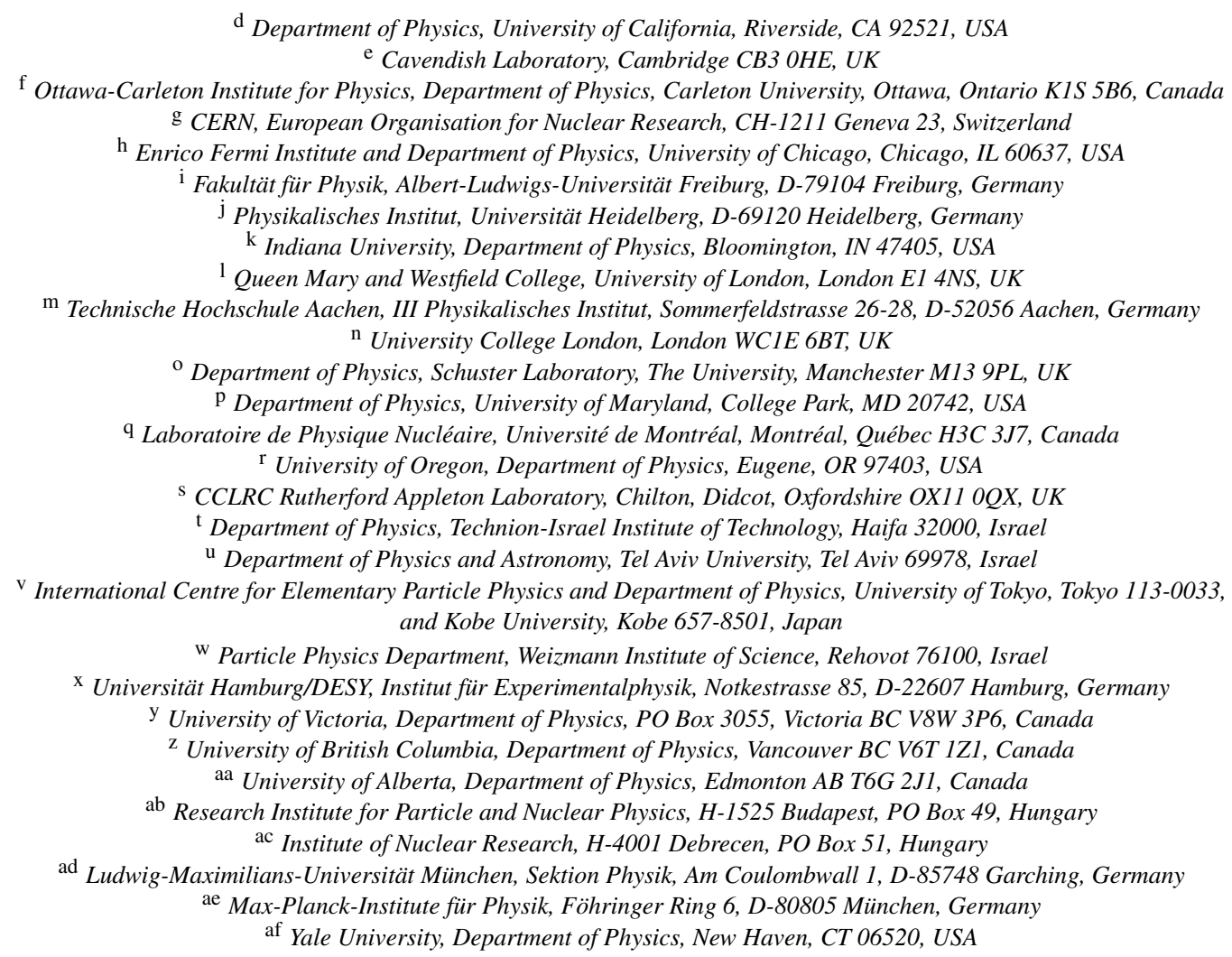

Received 13 October 2006; received in revised form 3 May 2007; accepted 4 June 2007

Available online 7 June 2007

Editor: L. Rolandi

\begin{abstract}
The inclusive production of charged hadrons in the collisions of quasi-real photons $\left(\mathrm{e}^{+} \mathrm{e}^{-} \rightarrow \mathrm{e}^{+} \mathrm{e}^{-}+\mathrm{X}\right)$ has been measured using the OPAL detector at LEP. The data were taken at $\mathrm{e}^{+} \mathrm{e}^{-}$centre-of-mass energies from 183 to $209 \mathrm{GeV}$. The differential cross-sections as a function of the transverse momentum and the pseudorapidity of the hadrons are compared to theoretical calculations of up to next-to-leading order (NLO) in the strong coupling constant $\alpha_{s}$. The data are also compared to a measurement by the L3 Collaboration, in which a large deviation from the NLO predictions is observed.
\end{abstract}

(C) 2007 Elsevier B.V. All rights reserved.

\footnotetext{
* Corresponding author.

E-mail address: plane@cern.ch (D.E. Plane).

1 Now at The University of Melbourne, Victoria, Australia.

2 Now at University of Antwerpen, Physics Department, B-2610 Antwerpen, Belgium; supported by Interuniversity Attraction Poles Programme-Belgian Science Policy.

3 And Institute of Nuclear Research, Debrecen, Hungary.

4 Now at Department of Physics, University of Illinois at Urbana-Champaign, USA.

5 And High Energy Accelerator Research Organisation (KEK), Tsukuba, Ibaraki, Japan.

6 Now at Technische Universität, Dresden, Germany.

7 And Department of Experimental Physics, University of Debrecen, Hungary.

8 Now at University of Toronto, Dept of Physics, Toronto, Canada.

9 Now at University of California, San Diego, USA.

10 Now at University of Alberta.
}

11 Current address Bergische Universität, Wuppertal, Germany.

12 And at TRIUMF, Vancouver, Canada V6T 2A3.

13 Now at University of Pennsylvania, Philadelphia, Pennsylvania, USA.

14 And Research Institute for Particle and Nuclear Physics, Budapest, Hungary.

15 Now at University of Mining and Metallurgy, Cracow, Poland.

16 Now at IEKP Universität Karlsruhe, Germany.

17 And Manchester University Manchester, M13 9PL, United Kingdom.

18 Now at DESY.

19 And MPI München.

20 Now at University of Liverpool, Dept of Physics, Liverpool L69 3BX, UK.

21 Now at CERN.

22 Now at DESY Zeuthen.

23 Now at Institute of Physics, Academy of Sciences of the Czech Republic 18221 Prague, Czech Republic.

24 Now at TRIUMF, Vancouver, Canada.

25 Now at IPHE Université de Lausanne, CH-1015 Lausanne, Switzerland. 


\section{Introduction}

Hadronic interactions of two photons lead to the production of hadrons whose properties in Quantum Chromodynamics (QCD) depend on the underlying partonic processes, as well as on the way in which the partons are transformed into observable hadrons. The study of inclusive hadron production is therefore an appropriate tool to investigate the validity of QCD in hadronic photon-photon interactions. Furthermore a drastic discrepancy between data and NLO QCD has recently been observed in this process [1] which would indicate the breakdown of this otherwise so successful theory and hence requires further study. Our measurements complement similar studies of jet production $[2,3]$. The same partonic processes are at work in QCD in both cases, but hadronisation is usually treated differently. To predict the production of single hadrons, universal fragmentation functions obtained from fits to independent data are utilised to describe the transition from partons to hadrons. Jet observables on the other hand are designed to keep the influence of hadronisation processes small. The remaining distortion with respect to partonic jet observables is estimated using hadronisation models. The reliable calculation of an observable in perturbative QCD requires a sufficiently high energy scale which in our case is provided by the transverse momenta of the charged particles. First results have been published by OPAL at $\mathrm{e}^{+} \mathrm{e}^{-}$centre-of-mass energies $\sqrt{s_{\mathrm{ee}}}=161$ and $172 \mathrm{GeV}$ [4]. L3 has published results in the range $\sqrt{s_{\mathrm{ee}}}=189-202 \mathrm{GeV}$ [1]. This Letter extends the OPAL measurements of the transverse momentum spectra and pseudorapidity ${ }^{27}$ distributions of charged hadrons in [4] using data taken at $\mathrm{e}^{+} \mathrm{e}^{-}$centre-of-mass energies from $\sqrt{s_{\text {ee }}}=183$ to $209 \mathrm{GeV}$, representing a roughly thirty-fold increase in integrated luminosity.

Hadronic photon-photon interactions can be modelled by assuming that the photons can either interact directly via a quark propagator or appear resolved through fluctuations into hadronic states. In leading order QCD this model leads to three different event classes for $\gamma \gamma$ interactions: direct, singleresolved and double-resolved, where resolved means that the partons (quarks or gluons) inside the hadronic photon take part in the hard interaction. The probability to find partons in the photon is parametrised by parton density functions.

At LEP the photons are radiated by the beam electrons ${ }^{28}$ and carry mostly small negative four-momenta squared, $Q^{2}$. In this Letter events are considered only if the electrons are scattered at small angles and are not detected. Both photons are therefore quasi-real $\left(Q^{2} \approx 0 \mathrm{GeV}^{2}\right)$. Differential hadron production cross-sections are measured as a function of the transverse mo-

\footnotetext{
26 Now at University of Kansas, Dept of Physics and Astronomy, Lawrence, KS 66045, USA.

Deceased.

27 In the OPAL coordinate system the $x$ axis points towards the centre of the LEP ring, the $y$ axis points upwards and the $z$ axis points in the direction of the electron beam. The polar angle $\theta$, the azimuthal angle $\phi$ and the radius $r$ denote the usual spherical coordinates. The pseudorapidity $\eta$ is defined as $\eta=-\ln \tan (\theta / 2)$.

28 Positrons are also referred to as electrons.
}

mentum and the pseudorapidity of charged hadrons. The inclusive cross-sections are compared to Monte Carlo (MC) event generators in leading order $\alpha_{s}$ and theoretical calculations in next-to-leading order (NLO) $\alpha_{s}$ for this process.

\section{The OPAL detector}

A detailed description of the OPAL detector can be found elsewhere [5]. The central tracking was performed inside a solenoidal magnet which provided a uniform axial magnetic field of $0.435 \mathrm{~T}$ along the beam axis. Starting with the innermost components, the tracking system consisted of a high precision silicon microvertex detector, a precision vertex drift chamber, a large volume jet chamber with 159 layers of axial anode wires and a set of $z$ chambers measuring the track coordinates along the beam direction. The transverse momenta, $p_{\mathrm{T}}$, of tracks are measured with a precision parametrised by $\sigma_{p_{\mathrm{T}}} / p_{\mathrm{T}}=\sqrt{0.02^{2}+\left(0.0015 \cdot p_{\mathrm{T}}\right)^{2}}\left(p_{\mathrm{T}}\right.$ in $\left.\mathrm{GeV}\right)$ in the central region $|\cos \theta|<0.73$. In this Letter transverse is always defined with respect to the beam axis.

The magnet was surrounded in the barrel region $(|\cos \theta|<$ 0.82 ) by a lead glass electromagnetic calorimeter (ECAL) and a hadronic sampling calorimeter (HCAL), which in turn were surrounded by muon chambers. Similar layers of detectors were installed in the endcaps $(0.82<|\cos \theta|<0.98)$. The small angle region from 47 to $140 \mathrm{mrad}$ around the beam pipe on both sides of the interaction point was covered by the forward calorimeters (FD) and the region from 33 to 59 mrad by the silicon tungsten luminometers (SW). The latter were used to determine the luminosity by counting small-angle Bhabha scattering events.

\section{Kinematics and Monte Carlo simulation}

The properties of the two interacting photons $(i=1,2)$ are described by their negative four-momentum transfers $Q_{i}^{2}$ and their invariant mass. Each $Q_{i}^{2}$ is related to the electron scattering angle $\theta_{i}^{\prime}$ relative to the beam direction by

$$
Q_{i}^{2}=-\left(p_{i}-p_{i}^{\prime}\right)^{2} \approx 2 E_{i} E_{i}^{\prime}\left(1-\cos \theta_{i}^{\prime}\right),
$$

where $p_{i}$ and $p_{i}^{\prime}$ are the four-momenta of the beam electron and the scattered electron, respectively, and $E_{i}$ and $E_{i}^{\prime}$ are their energies. Events with detected scattered electrons (single-tagged or double-tagged events) are excluded from the analysis. Driven by the angular acceptance of the FD and SW calorimeters a value of $Q^{2}=4.5 \mathrm{GeV}^{2}$ is used in this analysis to separate the quasi-real photons of untagged events from the virtual photons of tagged events. The median $Q^{2}$ resulting from this definition cannot be determined from data since the scattered electrons are not tagged. For the kinematic range of this analysis the MC simulations predict the median $Q^{2}$ to be of the order of $10^{-4} \mathrm{GeV}^{2}$.

The hadronic invariant mass of the photon-photon system, $W$, can be obtained from the energies and momenta $\left(E_{\mathrm{h}}, \vec{p}_{\mathrm{h}}\right)$ of final state particles:

$$
W^{2}=\left(\sum_{\mathrm{h}} E_{\mathrm{h}}\right)^{2}-\left(\sum_{\mathrm{h}} \vec{p}_{\mathrm{h}}\right)^{2} \text {. }
$$


The MC generators PYTHIA 5.722 [6] and PHOJET 1.10 [7] have been used to simulate photon-photon interactions. PYTHIA uses the SaS-1D parametrisation [8] for the photon parton densities and PHOJET uses the GRV parametrisation [9].

All relevant background processes were studied using MC generators. Multihadronic events $\left(\mathrm{e}^{+} \mathrm{e}^{-} \rightarrow \mathrm{q} \overline{\mathrm{q}}(\gamma)\right)$ were simulated with PYTHIA 6.125. KORALZ 4.02 [10] was used to generate the process $\mathrm{e}^{+} \mathrm{e}^{-} \rightarrow \tau^{+} \tau^{-}(\gamma)$ and VERMASEREN [11] to generate $\mathrm{e}^{+} \mathrm{e}^{-} \rightarrow \mathrm{e}^{+} \mathrm{e}^{-} \tau^{+} \tau^{-}$. Deep-inelastic e $\gamma$ events were simulated with HERWIG 5.9 [12].

All signal and background MC samples were generated with full simulation of the OPAL detector [13]. They are analysed using the same reconstruction algorithms as for the data. The number of generated $\mathrm{MC}$ events corresponds to ten times the data luminosity, with the exception of $\mathrm{e}^{+} \mathrm{e}^{-} \rightarrow \mathrm{e}^{+} \mathrm{e}^{-} \tau^{+} \tau^{-}$, for which three times the data luminosity was available.

\section{Event selection}

The production of charged hadrons was studied using data taken at $\mathrm{e}^{+} \mathrm{e}^{-}$centre-of-mass energies, $\sqrt{s_{\mathrm{ee}}}$, from 183 to $209 \mathrm{GeV}$, amounting to a total integrated luminosity of 612.8 $\mathrm{pb}^{-1}$. The luminosity weighted average centre-of-mass energy is $195.8 \mathrm{GeV}$. Two-photon events are selected with the following set of cuts:

- At least six tracks must have been found in the tracking chambers. A track is required to have a minimum transverse momentum of $120 \mathrm{MeV}$ with respect to the $z$ axis and at least 40 hits in the central jet chamber. The number of measured hits in the jet chamber must be more than half of the number of possible hits given the track direction. The radial distance of nearest approach of the track to the primary vertex has to be less than $0.15 \mathrm{~cm}$.

- The visible invariant hadronic mass calculated from the position and the energy of the clusters measured in the ECAL has to be greater than $3 \mathrm{GeV}$.

- The sum of all energy deposits in the ECAL and the HCAL has to be less than $50 \mathrm{GeV}$ to remove background from hadronic $\mathrm{Z}$ decays in events with a radiative return to the $\mathrm{Z}$ peak.

- The missing transverse momentum of the event measured in the ECAL and the FD has to be less than $8 \mathrm{GeV}$.

- To reject events with scattered electrons in the FD or SW, the total energy sum measured in the FD and SW has to be less than $60 \mathrm{GeV}$.

- The background due to beam-gas or beam-wall interactions is reduced by requiring the radial distance of the primary vertex from the beam axis to be less than $2 \mathrm{~cm}$ and the distance from the nominal vertex position along the $z$ direction to be less than $3 \mathrm{~cm}$.

After all cuts the data sample contains 1144035 events. The main remaining background processes are multihadronic $\mathrm{Z}$ decays, $\mathrm{e}^{+} \mathrm{e}^{-} \rightarrow \mathrm{e}^{+} \mathrm{e}^{-} \tau^{+} \tau^{-}$and deep-inelastic e $\gamma$ scattering. Other background processes are found to be negligible.
The multihadronic background is mainly reduced by the cut on the sum of the energy measured by the ECAL and the HCAL and by the cut on the missing transverse momentum. The $\mathrm{e}^{+} \mathrm{e}^{-} \rightarrow \mathrm{e}^{+} \mathrm{e}^{-} \tau^{+} \tau^{-}$processes are reduced by the cuts on the number of tracks and deep-inelastic e $\gamma$ events are rejected by the cut on the energy in SW and FD. The trigger efficiency for events in this region of phase space is close to 100\% [4] and no correction is applied.

From the MC simulations it is estimated that after all cuts the total remaining background is below $2 \%$ overall, but increasing to up to $50 \%$ at very high transverse momenta of the charged particles. The background is subtracted bin-by-bin from the distributions measured before corrections for detector resolution and acceptance are applied. The signal MC generators PHOJET and PYTHIA are found to underestimate the cross-section. In particular they show a shape different from that in the data of the $p_{\mathrm{T}}$-distribution of charged hadrons. To study a potential bias resulting from this deficiency the MC events have been reweighted by a suitable $p_{\mathrm{T}}$-dependent function to resemble the data. Both the original and the reweighted MC distributions are used in the analysis and any differences seen are included in the systematic uncertainty.

\section{Analysis}

Only particles with a lifetime $\tau>0.3 \mathrm{~ns}$ are used to define the primary charged hadronic multiplicity. The primary charged hadrons originate either directly from the primary interaction or from the decay of particles with a lifetime $\tau<0.3 \mathrm{~ns}$. The decay products of $\Lambda$ and $\mathrm{K}_{\mathrm{S}}^{0}$ particles are hence considered as primary hadrons in this analysis. The measured transverse momentum and pseudorapidity distributions of charged hadrons have to be corrected for the losses due to event and track selection cuts, the acceptance and the resolution of the detector. This is done using the MC events which were processed by the full detector simulation and reconstruction chain. The data are corrected by multiplying the experimental distribution, e.g. of the transverse momentum $p_{\mathrm{T}}$, with correction factors which are calculated as the bin-by-bin ratio of the generated and the reconstructed MC distributions:

$\left(\frac{\mathrm{d} \sigma}{\mathrm{d} p_{\mathrm{T}}}\right)_{\text {corrected }}=\frac{\left(\frac{\mathrm{d} \sigma}{\mathrm{d} p_{\mathrm{T}}}\right)_{\text {generated }}^{\mathrm{MC}}}{\left(\frac{\mathrm{d} \sigma}{\mathrm{d} p_{\mathrm{T}}}\right)_{\text {reconstructed }}^{\mathrm{MC}}}\left(\frac{\mathrm{d} \sigma}{\mathrm{d} p_{\mathrm{T}}}\right)_{\text {measured }}$.

The ratio is calculated using both PYTHIA and PHOJET, with the mean value used to correct the data. The pseudorapidity distributions are corrected in the same way. This method only yields reliable results if migration effects between bins due to the finite resolution of the measurement are small. The bins of the $p_{\mathrm{T}}$ and $|\eta|$ distributions have therefore been chosen to be significantly larger than the resolution expected from the MC simulation. To avoid regions where the detector has little or no acceptance, all measurements of charged hadron distributions were restricted to the range $|\eta|<1.5$. The average transverse momentum $\left\langle p_{\mathrm{T}}\right\rangle$ and the average pseudorapidity $\langle|\eta|\rangle$ in each bin is calculated directly from the data since detector corrections are small compared to the statistical uncertainties. 
In order to be able to use Eq. (3) for the detector correction in each bin of $W$, the bins in $W$ must be larger than the experimental resolution and the average reconstructed hadronic invariant mass, $\left\langle W_{\text {rec }}\right\rangle$, should be approximately equal to the average generated hadronic invariant mass, $\left\langle W_{\text {gen }}\right\rangle$. In this case bin-tobin migrations are minimised. The visible invariant mass, $W_{\text {vis }}$, is determined from all tracks and calorimeter clusters, including the forward detectors and the silicon tungsten calorimeters, after applying a matching algorithm to avoid double counting of particle momenta [15].

The ratio of $W_{\text {gen }}$ and $W_{\text {vis }}$ depends on the event kinematics, and was therefore determined separately in two distinct regions of phase space. The discriminating variable chosen for this purpose is $p_{\mathrm{T}, \max }$, the highest transverse momentum of any track in the event. The average visible hadronic invariant mass, $\left\langle W_{\text {vis }}\right\rangle$, and the resolution on $W_{\text {vis }}$ as a function of the generated hadronic invariant mass $W_{\text {gen }}$ for events with $p_{\mathrm{T} \text {, max }}$ smaller and larger than $5 \mathrm{GeV}$ are shown in Fig. 1(a) and (c). The polynomial fits superimposed on Fig. 1(b) and (d) are used as a correction function so that $\left\langle W_{\text {gen }}\right\rangle /\left\langle W_{\text {rec }}\right\rangle \approx 1$.

The distributions are measured for invariant masses $10<$ $W<30 \mathrm{GeV}, 30<W<50 \mathrm{GeV}, 50<W<125 \mathrm{GeV}$ and $10<W<125 \mathrm{GeV}$ where $W$ represents the hadronic invariant mass after correcting for detector effects. To facilitate comparisons to the results in [1], the ranges $W>30 \mathrm{GeV}$ and $W>50 \mathrm{GeV}$ are also considered.

\section{Systematic uncertainties}

The following sources of systematic uncertainties have been investigated:

- Significant amounts of background need to be subtracted from the data at large $p_{\mathrm{T}}$, where the dominant source of background is multihadronic events $\left(\mathrm{e}^{+} \mathrm{e}^{-} \rightarrow \mathrm{q} \overline{\mathrm{q}}(\gamma)\right)$. The amount of this background subtracted from the data is varied by $\pm 10 \%$ to estimate the uncertainty associated to this procedure. The amount by which the background must be varied is estimated from studying the MC description of the data in regions of the phase space where the background dominates. The effect on the measured cross-section is usually $1 \%$ or less for low transverse momenta, increasing to up to $7 \%$ at the highest momenta measured.

- The selection criteria described in Section 4 are varied simultaneously both to be more restrictive and to allow more events into the analysis to exclude a strong dependence on the event selection. Selection criteria based on energy measurements are varied by $5 \%$ in the ECAL and HCAL, and by $10 \%$ in the FD and SW calorimeters. The number of tracks required is changed by \pm 1 . The allowed radial distance of the tracks is varied by $5 \%$. The uncertainty on the cross-section derived from all these variations is typically 1-6\%.
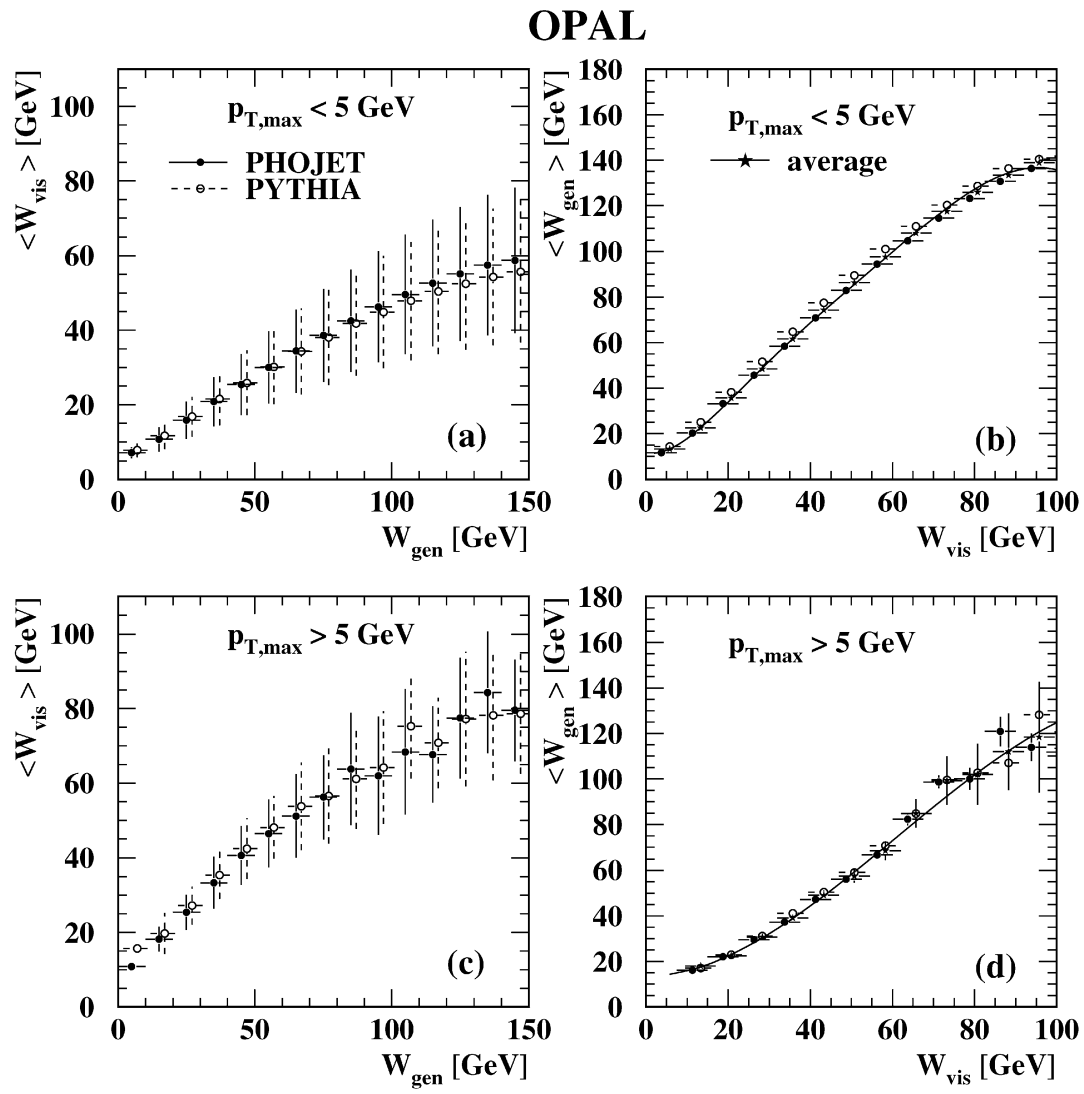

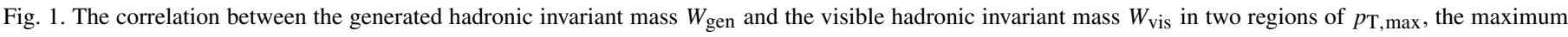

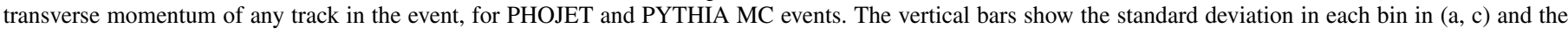

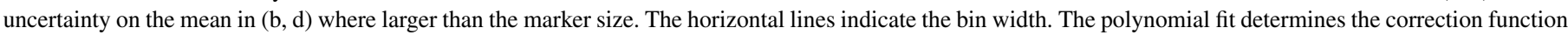
for $W_{\text {vis }}$. 


\section{OPAL}
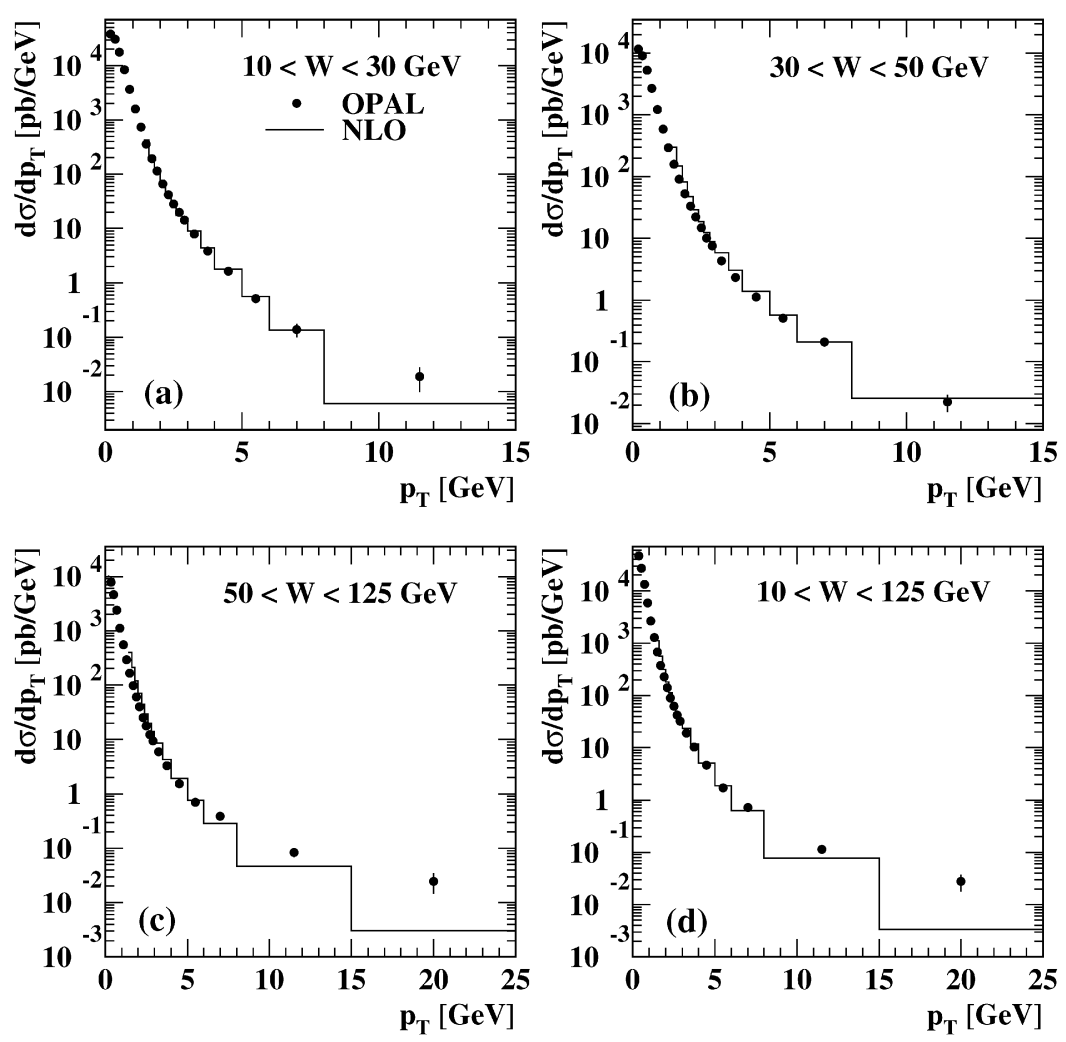

Fig. 2. Differential inclusive charged hadron production cross-sections, $\mathrm{d} \sigma / \mathrm{d} p_{\mathrm{T}}$, for $|\eta|<1.5$ in the $W$ ranges (a) $10<W<30 \mathrm{GeV}$; (b) $30<W<50 \mathrm{GeV}$; (c) $50<W<125 \mathrm{GeV}$ and (d) $10<W<125 \mathrm{GeV}$. The error bars show the statistical and systematic uncertainties added in quadrature when larger than the marker, which indicates the bin centre. The data are compared to an NLO calculation [16].

- Both PHOJET and PYTHIA have been used to calculate the correction factors applied to the data. The resulting distributions are averaged and the difference between the two distributions is used to define the systematic uncertainty. The distributions obtained from both programs have been reweighted for a better description of the data. The difference between using the reweighted and the original MC distributions to calculate the correction factors has been included in the systematic uncertainty. The uncertainty derived from this study is below $5 \%$.

- The systematic uncertainty due to the energy scale of the ECAL for the range of energies in this analysis was estimated by varying the reconstructed ECAL energy in the MC by $\pm 3 \%$ [14]. The cross-sections change by up to $4 \%$ due to this variation.

- Studying vertex and net charge distributions it is estimated that about $2 \%$ of the selected events are due to beam-gas or beam-wall interactions. This remaining background is treated as a systematic uncertainty of $2 \%$.

Systematic uncertainties due to the modelling of the detector resolution for the measurement of tracks [4] and due to the luminosity measurement were found to be negligible. The total systematic uncertainty was obtained by adding all systematic uncertainties in quadrature.

\section{Results and conclusions}

The differential inclusive cross-sections $\mathrm{d} \sigma / \mathrm{d} p_{\mathrm{T}}$ for charged hadrons in four ranges of the hadronic invariant mass $W$ are shown in Fig. 2 and are given in Tables 1 and 2. The data points in the figure indicate the bin centre. Both data and calculations are presented for quasi-real photons of virtualities $Q^{2}<4.5 \mathrm{GeV}^{2}$, as described in Section 3 .

Calculations in NLO QCD [16] are compared to the data. The cross-sections are calculated using the QCD partonic cross-sections in NLO for direct, single- and double-resolved processes. The hadronic cross-section is a convolution of the Weizsäcker-Williams effective photon distribution, the parton distribution functions and the fragmentation functions of [17]. The AFG-HO parametrisation of the parton densities of the photon [18] is used with $\Lambda_{\overline{M S}}^{5}=221 \mathrm{MeV}$. The renormalisation and factorisation scales in the calculation are set equal to $p_{\mathrm{T}}$. The cross-section calculation was repeated for the kinematic conditions of the present analysis. For the differential cross-section $\mathrm{d} \sigma / \mathrm{d} p_{\mathrm{T}}$ a minimum $p_{\mathrm{T}}$ of $1.5 \mathrm{GeV}$ is required to ensure the validity of the perturbative QCD calculation. Even at $p_{\mathrm{T}}=1.5 \mathrm{GeV}$ the cross-sections change by up to $80 \%$ when varying the renormalisation and factorisation scales by factors of two. This uncertainty decreases rapidly to between $10 \%$ and $15 \%$ for $p_{\mathrm{T}}=3.5 \mathrm{GeV}$ and above. The differential cross- 
Table 1

Differential inclusive charged hadron production cross-sections $\mathrm{d} \sigma / \mathrm{d} p_{\mathrm{T}}$ for $|\eta|<1.5$ and in the $W$ ranges $10<W<30 \mathrm{GeV}$ and $30<W<50 \mathrm{GeV}$. The first uncertainty is the statistical uncertainty and the second uncertainty is the systematic uncertainty. No value is given if the error on $\left\langle p_{\mathrm{T}}\right\rangle$ is less than 0.01

\begin{tabular}{|c|c|c|c|c|}
\hline \multirow[b]{2}{*}{$p_{\mathrm{T}}[\mathrm{GeV}]$} & \multicolumn{2}{|c|}{$10<W<30 \mathrm{GeV}$} & \multicolumn{2}{|c|}{$30<W<50 \mathrm{GeV}$} \\
\hline & $\left\langle p_{\mathrm{T}}\right\rangle[\mathrm{GeV}]$ & $\mathrm{d} \sigma / \mathrm{d} p_{\mathrm{T}}[\mathrm{pb} / \mathrm{GeV}]$ & $\left\langle p_{\mathrm{T}}\right\rangle[\mathrm{GeV}]$ & $\mathrm{d} \sigma / \mathrm{d} p_{\mathrm{T}}[\mathrm{pb} / \mathrm{GeV}]$ \\
\hline $0.12-0.28$ & 0.20 & $(3.78 \pm 0.01 \pm 0.18) \times 10^{4}$ & 0.20 & $(1.15 \pm 0.00 \pm 0.03) \times 10^{4}$ \\
\hline $0.28-0.44$ & 0.35 & $(3.01 \pm 0.01 \pm 0.13) \times 10^{4}$ & 0.35 & $(9.01 \pm 0.02 \pm 0.21) \times 10^{3}$ \\
\hline $0.44-0.60$ & 0.51 & $(1.74 \pm 0.00 \pm 0.06) \times 10^{4}$ & 0.51 & $(5.26 \pm 0.02 \pm 0.13) \times 10^{3}$ \\
\hline $0.60-0.80$ & 0.69 & $(8.43 \pm 0.03 \pm 0.29) \times 10^{3}$ & 0.69 & $(2.69 \pm 0.01 \pm 0.07) \times 10^{3}$ \\
\hline $0.80-1.00$ & 0.89 & $(3.62 \pm 0.02 \pm 0.16) \times 10^{3}$ & 0.89 & $(1.22 \pm 0.01 \pm 0.03) \times 10^{3}$ \\
\hline $1.00-1.20$ & 1.09 & $(1.58 \pm 0.01 \pm 0.09) \times 10^{3}$ & 1.09 & $(5.85 \pm 0.05 \pm 0.19) \times 10^{2}$ \\
\hline $1.20-1.40$ & 1.29 & $(7.29 \pm 0.08 \pm 0.48) \times 10^{2}$ & 1.29 & $(2.94 \pm 0.04 \pm 0.11) \times 10^{2}$ \\
\hline $1.40-1.60$ & 1.49 & $(3.61 \pm 0.06 \pm 0.25) \times 10^{2}$ & 1.49 & $(1.59 \pm 0.03 \pm 0.06) \times 10^{2}$ \\
\hline $1.60-1.80$ & 1.69 & $(1.92 \pm 0.04 \pm 0.13) \times 10^{2}$ & 1.69 & $(9.13 \pm 0.21 \pm 0.32) \times 10^{1}$ \\
\hline $1.80-2.00$ & 1.89 & $(1.13 \pm 0.03 \pm 0.08) \times 10^{2}$ & 1.89 & $(5.32 \pm 0.15 \pm 0.19) \times 10^{1}$ \\
\hline $2.00-2.20$ & 2.09 & $(6.68 \pm 0.23 \pm 0.44) \times 10^{1}$ & 2.09 & $(3.34 \pm 0.12 \pm 0.11) \times 10^{1}$ \\
\hline $2.20-2.40$ & 2.29 & $(4.17 \pm 0.19 \pm 0.26) \times 10^{1}$ & 2.29 & $(2.22 \pm 0.10 \pm 0.07) \times 10^{1}$ \\
\hline $2.40-2.60$ & 2.50 & $(2.85 \pm 0.16 \pm 0.17) \times 10^{1}$ & 2.50 & $(1.47 \pm 0.08 \pm 0.04) \times 10^{1}$ \\
\hline $2.60-2.80$ & 2.70 & $(1.98 \pm 0.13 \pm 0.12) \times 10^{1}$ & 2.70 & $(1.00 \pm 0.06 \pm 0.03) \times 10^{1}$ \\
\hline $2.80-3.00$ & 2.90 & $(1.45 \pm 0.12 \pm 0.09) \times 10^{1}$ & 2.90 & $(7.48 \pm 0.54 \pm 0.26) \times 10^{0}$ \\
\hline $3.00-3.50$ & 3.21 & $(7.93 \pm 0.56 \pm 0.50) \times 10^{0}$ & 3.23 & $(4.33 \pm 0.26 \pm 0.15) \times 10^{0}$ \\
\hline $3.50-4.00$ & 3.71 & $(3.87 \pm 0.42 \pm 0.27) \times 10^{0}$ & 3.73 & $(2.32 \pm 0.19 \pm 0.08) \times 10^{0}$ \\
\hline $4.00-5.00$ & 4.40 & $(1.63 \pm 0.22 \pm 0.15) \times 10^{0}$ & 4.42 & $(1.12 \pm 0.09 \pm 0.04) \times 10^{0}$ \\
\hline $5.00-6.00$ & 5.40 & $(5.16 \pm 0.78 \pm 0.55) \times 10^{-1}$ & 5.43 & $(5.05 \pm 0.66 \pm 0.19) \times 10^{-1}$ \\
\hline $6.00-8.00$ & $6.74 \pm 0.01$ & $(1.37 \pm 0.31 \pm 0.22) \times 10^{-1}$ & 6.74 & $(2.10 \pm 0.33 \pm 0.12) \times 10^{-1}$ \\
\hline $8.00-15.00$ & $9.52 \pm 0.12$ & $(1.90 \pm 0.86 \pm 0.33) \times 10^{-2}$ & $9.62 \pm 0.03$ & $(2.23 \pm 0.60 \pm 0.36) \times 10^{-2}$ \\
\hline
\end{tabular}

Table 2

Differential inclusive charged hadron production cross-sections $\mathrm{d} \sigma / \mathrm{d} p_{\mathrm{T}}$ for $|\eta|<1.5$ and in the $W$ ranges $50<W<125 \mathrm{GeV}$ and $10<W<125 \mathrm{GeV}$. The first uncertainty is the statistical uncertainty and the second uncertainty is the systematic uncertainty. No value is given if the error on $\left\langle p_{\mathrm{T}}\right\rangle$ is less than 0.01

\begin{tabular}{|c|c|c|c|c|}
\hline \multirow[b]{2}{*}{$p_{\mathrm{T}}[\mathrm{GeV}]$} & \multicolumn{2}{|c|}{$50<W<125 \mathrm{GeV}$} & \multicolumn{2}{|c|}{$10<W<125 \mathrm{GeV}$} \\
\hline & $\left\langle p_{\mathrm{T}}\right\rangle[\mathrm{GeV}]$ & $\mathrm{d} \sigma / \mathrm{d} p_{\mathrm{T}}[\mathrm{pb} / \mathrm{GeV}]$ & $\left\langle p_{\mathrm{T}}\right\rangle[\mathrm{GeV}]$ & $\mathrm{d} \sigma / \mathrm{d} p_{\mathrm{T}}[\mathrm{pb} / \mathrm{GeV}]$ \\
\hline $0.12-0.28$ & 0.20 & $(1.00 \pm 0.00 \pm 0.03) \times 10^{4}$ & 0.20 & $(5.93 \pm 0.00 \pm 0.21) \times 10^{4}$ \\
\hline $0.28-0.44$ & 0.35 & $(7.77 \pm 0.02 \pm 0.22) \times 10^{3}$ & 0.35 & $(4.68 \pm 0.00 \pm 0.18) \times 10^{4}$ \\
\hline $0.44-0.60$ & 0.51 & $(4.58 \pm 0.01 \pm 0.13) \times 10^{3}$ & 0.51 & $(2.71 \pm 0.00 \pm 0.10) \times 10^{4}$ \\
\hline $0.60-0.80$ & 0.69 & $(2.37 \pm 0.01 \pm 0.07) \times 10^{3}$ & 0.69 & $(1.34 \pm 0.00 \pm 0.05) \times 10^{4}$ \\
\hline $0.80-1.00$ & 0.89 & $(1.12 \pm 0.01 \pm 0.03) \times 10^{3}$ & 0.89 & $(5.87 \pm 0.01 \pm 0.25) \times 10^{3}$ \\
\hline $1.00-1.20$ & 1.09 & $(5.56 \pm 0.04 \pm 0.14) \times 10^{2}$ & 1.09 & $(2.68 \pm 0.01 \pm 0.14) \times 10^{3}$ \\
\hline $1.20-1.40$ & 1.29 & $(2.92 \pm 0.03 \pm 0.08) \times 10^{2}$ & 1.29 & $(1.29 \pm 0.01 \pm 0.07) \times 10^{3}$ \\
\hline $1.40-1.60$ & 1.49 & $(1.66 \pm 0.02 \pm 0.05) \times 10^{2}$ & 1.49 & $(6.78 \pm 0.04 \pm 0.38) \times 10^{2}$ \\
\hline $1.60-1.80$ & 1.69 & $(9.74 \pm 0.15 \pm 0.30) \times 10^{1}$ & 1.69 & $(3.78 \pm 0.03 \pm 0.20) \times 10^{2}$ \\
\hline $1.80-2.00$ & 1.89 & $(6.10 \pm 0.11 \pm 0.21) \times 10^{1}$ & 1.89 & $(2.27 \pm 0.02 \pm 0.12) \times 10^{2}$ \\
\hline $2.00-2.20$ & 2.09 & $(4.01 \pm 0.09 \pm 0.15) \times 10^{1}$ & 2.09 & $(1.41 \pm 0.02 \pm 0.07) \times 10^{2}$ \\
\hline $2.20-2.40$ & 2.29 & $(2.53 \pm 0.07 \pm 0.10) \times 10^{1}$ & 2.29 & $(9.03 \pm 0.16 \pm 0.44) \times 10^{1}$ \\
\hline $2.40-2.60$ & 2.50 & $(1.76 \pm 0.06 \pm 0.09) \times 10^{1}$ & 2.50 & $(6.18 \pm 0.13 \pm 0.31) \times 10^{1}$ \\
\hline $2.60-2.80$ & 2.70 & $(1.22 \pm 0.05 \pm 0.06) \times 10^{1}$ & 2.70 & $(4.26 \pm 0.11 \pm 0.22) \times 10^{1}$ \\
\hline $2.80-3.00$ & 2.90 & $(9.52 \pm 0.44 \pm 0.50) \times 10^{0}$ & 2.90 & $(3.25 \pm 0.09 \pm 0.17) \times 10^{1}$ \\
\hline $3.00-3.50$ & 3.23 & $(5.99 \pm 0.22 \pm 0.30) \times 10^{0}$ & 3.23 & $(1.93 \pm 0.05 \pm 0.10) \times 10^{1}$ \\
\hline $3.50-4.00$ & 3.73 & $(3.33 \pm 0.16 \pm 0.22) \times 10^{0}$ & 3.73 & $(1.03 \pm 0.03 \pm 0.06) \times 10^{1}$ \\
\hline $4.00-5.00$ & 4.40 & $(1.52 \pm 0.08 \pm 0.15) \times 10^{0}$ & 4.43 & $(4.66 \pm 0.16 \pm 0.34) \times 10^{0}$ \\
\hline $5.00-6.00$ & 5.43 & $(7.02 \pm 0.83 \pm 0.81) \times 10^{-1}$ & 5.43 & $(1.71 \pm 0.11 \pm 0.13) \times 10^{0}$ \\
\hline $6.00-8.00$ & 6.83 & $(3.89 \pm 0.45 \pm 0.49) \times 10^{-1}$ & 6.79 & $(7.28 \pm 0.53 \pm 0.62) \times 10^{-1}$ \\
\hline $8.00-15.00$ & $10.18 \pm 0.01$ & $(8.40 \pm 1.34 \pm 0.90) \times 10^{-2}$ & 10.00 & $(1.14 \pm 0.14 \pm 0.09) \times 10^{-1}$ \\
\hline $15.00-25.00$ & $18.26 \pm 0.09$ & $(2.46 \pm 0.96 \pm 0.26) \times 10^{-2}$ & $18.27 \pm 0.08$ & $(2.76 \pm 0.99 \pm 0.22) \times 10^{-2}$ \\
\hline
\end{tabular}

section $\mathrm{d} \sigma / \mathrm{d}|\eta|$ is hence restricted to the region $p_{\mathrm{T}}>3.5 \mathrm{GeV}$ to allow a meaningful comparison.

In Fig. 2 the NLO calculation lies significantly below the data for transverse momenta greater than about $10 \mathrm{GeV}$, which can be reached in the highest $W$ range only. The predictions of
PHOJET and PYTHIA at high $p_{\mathrm{T}}$ (not shown) are similar to the NLO calculation.

The differential cross-section is nearly independent of $|\eta|$ in the range measured as can be seen in Fig. 3. The data points in the figure indicate the bin centre. The cross-section values 


\section{OPAL}
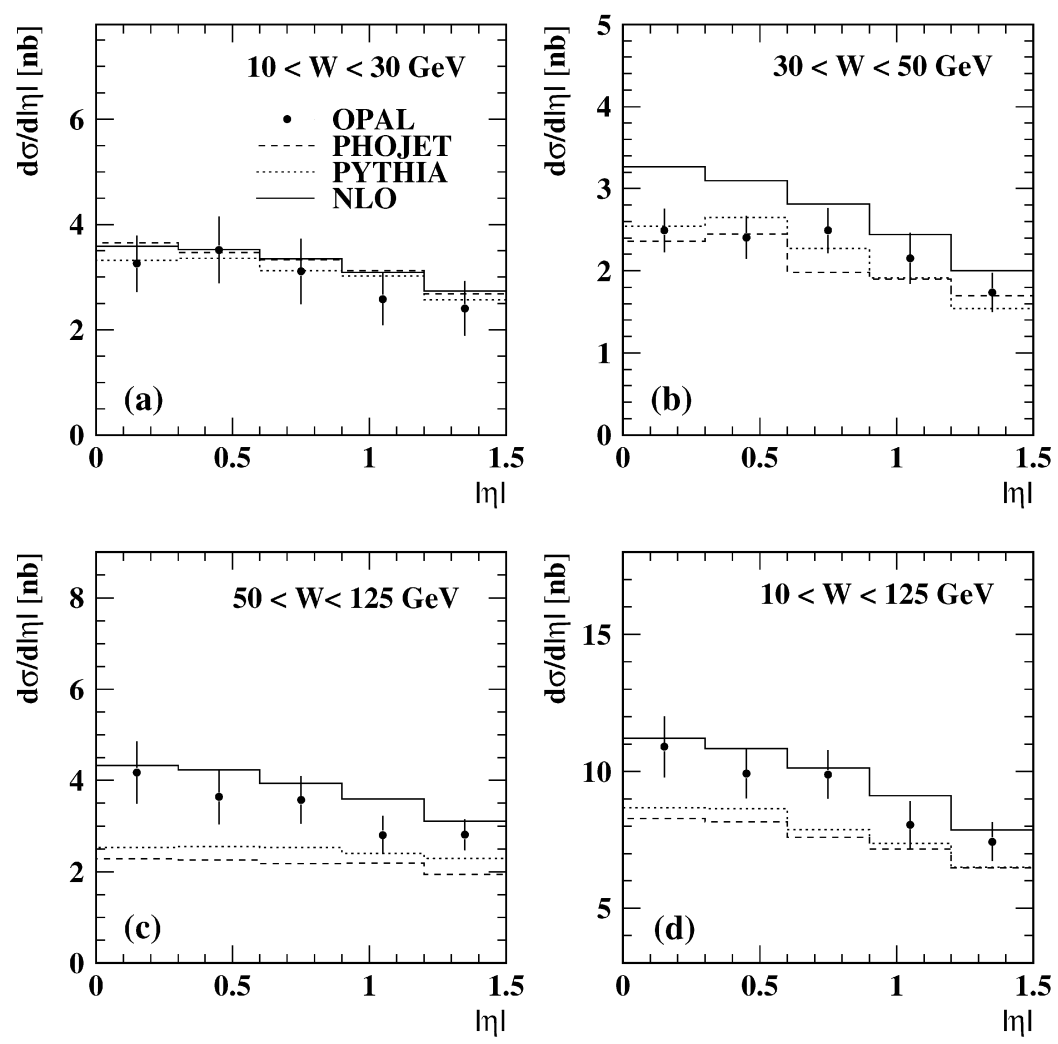

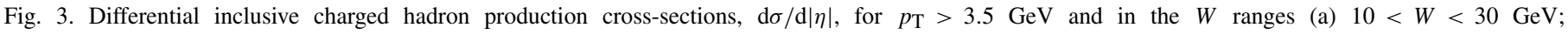

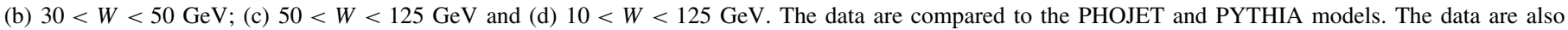

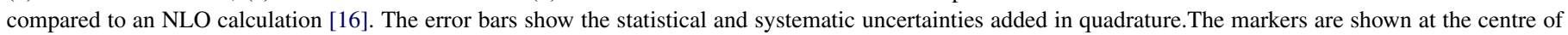
the bin.

Table 3

Differential inclusive charged hadron production cross-sections $\mathrm{d} \sigma / \mathrm{d}|\eta|$ for $p_{\mathrm{T}}>3.5 \mathrm{GeV}$ and in the $W$ ranges $10<W<30 \mathrm{GeV}, 30<W<50 \mathrm{GeV}, 50<W<$ $125 \mathrm{GeV}$ and $10<W<125 \mathrm{GeV}$. The first uncertainty is the statistical uncertainty and the second uncertainty is the systematic uncertainty

\begin{tabular}{|c|c|c|c|c|}
\hline \multirow[b]{2}{*}{$|\eta|$} & \multicolumn{2}{|c|}{$10<W<30 \mathrm{GeV}$} & \multicolumn{2}{|c|}{$30<W<50 \mathrm{GeV}$} \\
\hline & $\langle|\eta|\rangle$ & $\mathrm{d} \sigma / \mathrm{d}|\eta|[\mathrm{pb}]$ & $\langle|\eta|\rangle$ & $\mathrm{d} \sigma / \mathrm{d}|\eta|[\mathrm{pb}]$ \\
\hline $0.0-0.3$ & 0.149 & $3.26 \pm 0.45 \pm 0.29$ & 0.151 & $2.49 \pm 0.26 \pm 0.07$ \\
\hline $0.3-0.6$ & 0.445 & $3.52 \pm 0.50 \pm 0.39$ & 0.451 & $2.41 \pm 0.25 \pm 0.08$ \\
\hline $0.6-0.9$ & 0.754 & $3.11 \pm 0.47 \pm 0.41$ & 0.759 & $2.48 \pm 0.26 \pm 0.07$ \\
\hline $0.9-1.2$ & 1.047 & $2.58 \pm 0.43 \pm 0.24$ & 1.049 & $2.15 \pm 0.26 \pm 0.17$ \\
\hline \multirow[t]{2}{*}{$1.2-1.5$} & 1.348 & $2.40 \pm 0.44 \pm 0.28$ & 1.341 & $1.74 \pm 0.23 \pm 0.07$ \\
\hline & \multicolumn{2}{|c|}{$50<W<125 \mathrm{GeV}$} & \multicolumn{2}{|c|}{$10<W<125 \mathrm{GeV}$} \\
\hline$|\eta|$ & $\langle|\eta|\rangle$ & $\mathrm{d} \sigma / \mathrm{d}|\eta|[\mathrm{pb}]$ & $\langle|\eta|\rangle$ & $\mathrm{d} \sigma / \mathrm{d}|\eta|[\mathrm{pb}]$ \\
\hline $0.0-0.3$ & 0.149 & $4.17 \pm 0.27 \pm 0.63$ & 0.149 & $10.90 \pm 0.65 \pm 0.90$ \\
\hline $0.3-0.6$ & 0.449 & $3.63 \pm 0.25 \pm 0.53$ & 0.448 & $9.92 \pm 0.62 \pm 0.65$ \\
\hline $0.6-0.9$ & 0.759 & $3.57 \pm 0.25 \pm 0.46$ & 0.757 & $9.89 \pm 0.63 \pm 0.63$ \\
\hline $0.9-1.2$ & 1.048 & $2.81 \pm 0.23 \pm 0.34$ & 1.047 & $8.04 \pm 0.58 \pm 0.67$ \\
\hline $1.2-1.5$ & 1.345 & $2.81 \pm 0.22 \pm 0.25$ & 1.345 & $7.42 \pm 0.55 \pm 0.44$ \\
\hline
\end{tabular}

are given in Table 3 . The NLO calculation reproduces the data well within the uncertainties of the calculation. PYTHIA and PHOJET describe the shape of the distributions correctly for all regions of the phase space measured, but are below the data in normalisation for large values of $W$.

Fig. 4(a) and (b) and Table 4 show the differential crosssection $\mathrm{d} \sigma / \mathrm{d} p_{\mathrm{T}}$ for charged hadrons for $W>30 \mathrm{GeV}$ and $W>50 \mathrm{GeV}$ to facilitate a comparison with a recent mea- surement by L3 of charged pions in the pseudorapidity range $|\eta|<1.0$ [1]. The OPAL data shown in Fig. 4(c) and (d) and Table 5 have been scaled to account for the reduced $|\eta|$ range and for the fraction of charged pions of all charged hadrons using MC simulations. The OPAL data points in Fig. 4 are plotted at the mean transverse momentum across the bin, as done in the L3 publication. From this comparison it is evident that the distributions measured by OPAL fall more rapidly towards high 


\section{OPAL}
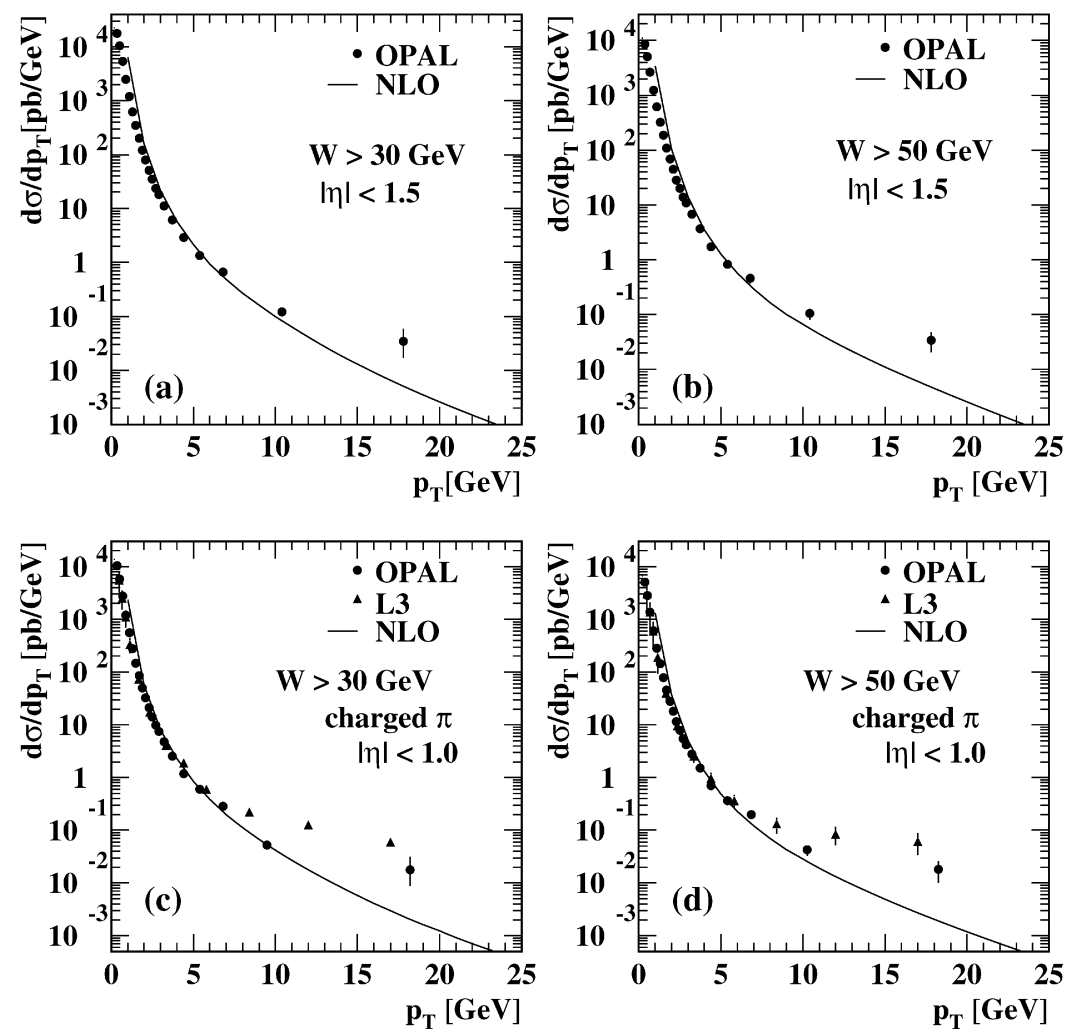

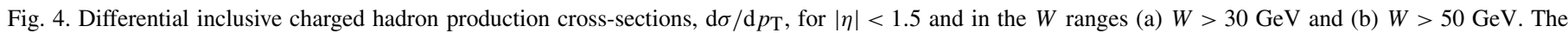

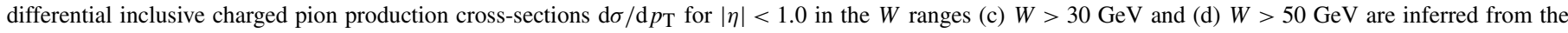

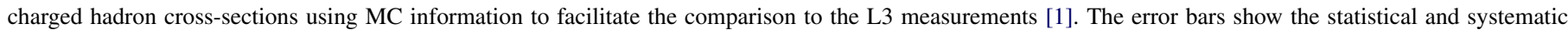

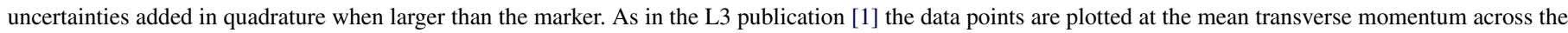
bin. The data are also compared to an NLO calculation [16].

Table 4

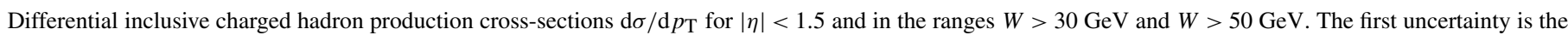
statistical uncertainty and the second uncertainty is the systematic uncertainty. No value is given if the error on $\left\langle p_{\mathrm{T}}\right\rangle$ is less than 0.01

\begin{tabular}{|c|c|c|c|c|}
\hline \multirow[b]{2}{*}{$p_{\mathrm{T}}[\mathrm{GeV}]$} & \multicolumn{2}{|l|}{$W>30 \mathrm{GeV}$} & \multicolumn{2}{|l|}{$W>50 \mathrm{GeV}$} \\
\hline & $\left\langle p_{\mathrm{T}}\right\rangle[\mathrm{GeV}]$ & $\mathrm{d} \sigma / \mathrm{d} p_{\mathrm{T}}[\mathrm{pb} / \mathrm{GeV}]$ & $\left\langle p_{\mathrm{T}}\right\rangle[\mathrm{GeV}]$ & $\mathrm{d} \sigma / \mathrm{d} p_{\mathrm{T}}[\mathrm{pb} / \mathrm{GeV}]$ \\
\hline $0.12-0.28$ & 0.20 & $(2.25 \pm 0.00 \pm 0.06) \times 10^{4}$ & 0.20 & $(1.10 \pm 0.00 \pm 0.04) \times 10^{4}$ \\
\hline $0.28-0.44$ & 0.35 & $(1.75 \pm 0.00 \pm 0.05) \times 10^{4}$ & 0.35 & $(8.50 \pm 0.02 \pm 0.28) \times 10^{3}$ \\
\hline $0.44-0.60$ & 0.51 & $(1.03 \pm 0.00 \pm 0.03) \times 10^{4}$ & 0.51 & $(5.02 \pm 0.02 \pm 0.16) \times 10^{3}$ \\
\hline $0.60-0.80$ & 0.69 & $(5.31 \pm 0.02 \pm 0.13) \times 10^{3}$ & 0.69 & $(2.61 \pm 0.01 \pm 0.08) \times 10^{3}$ \\
\hline $0.80-1.00$ & 0.89 & $(2.46 \pm 0.01 \pm 0.06) \times 10^{3}$ & 0.89 & $(1.24 \pm 0.01 \pm 0.04) \times 10^{3}$ \\
\hline $1.00-1.20$ & 1.09 & $(1.20 \pm 0.01 \pm 0.03) \times 10^{3}$ & 1.09 & $(6.17 \pm 0.05 \pm 0.18) \times 10^{2}$ \\
\hline $1.20-1.40$ & 1.29 & $(6.19 \pm 0.05 \pm 0.16) \times 10^{2}$ & 1.29 & $(3.25 \pm 0.04 \pm 0.09) \times 10^{2}$ \\
\hline $1.40-1.60$ & 1.49 & $(3.44 \pm 0.04 \pm 0.09) \times 10^{2}$ & 1.49 & $(1.85 \pm 0.03 \pm 0.05) \times 10^{2}$ \\
\hline $1.60-1.80$ & 1.69 & $(2.00 \pm 0.03 \pm 0.06) \times 10^{2}$ & 1.69 & $(1.09 \pm 0.02 \pm 0.03) \times 10^{2}$ \\
\hline $1.80-2.00$ & 1.89 & $(1.22 \pm 0.02 \pm 0.04) \times 10^{2}$ & 1.89 & $(6.87 \pm 0.18 \pm 0.20) \times 10^{1}$ \\
\hline $2.00-2.20$ & 2.09 & $(7.86 \pm 0.19 \pm 0.24) \times 10^{1}$ & 2.09 & $(4.51 \pm 0.14 \pm 0.14) \times 10^{1}$ \\
\hline $2.20-2.40$ & 2.29 & $(5.09 \pm 0.15 \pm 0.16) \times 10^{1}$ & 2.29 & $(2.86 \pm 0.11 \pm 0.10) \times 10^{1}$ \\
\hline $2.40-2.60$ & 2.50 & $(3.48 \pm 0.12 \pm 0.13) \times 10^{1}$ & 2.50 & $(2.01 \pm 0.09 \pm 0.08) \times 10^{1}$ \\
\hline $2.60-2.80$ & 2.70 & $(2.38 \pm 0.10 \pm 0.09) \times 10^{1}$ & 2.70 & $(1.38 \pm 0.08 \pm 0.06) \times 10^{1}$ \\
\hline $2.80-3.00$ & 2.90 & $(1.83 \pm 0.09 \pm 0.07) \times 10^{1}$ & 2.90 & $(1.08 \pm 0.07 \pm 0.05) \times 10^{1}$ \\
\hline $3.00-3.50$ & 3.23 & $(1.12 \pm 0.04 \pm 0.04) \times 10^{1}$ & 3.23 & $(6.78 \pm 0.34 \pm 0.27) \times 10^{0}$ \\
\hline $3.50-4.00$ & 3.73 & $(6.08 \pm 0.32 \pm 0.25) \times 10^{0}$ & 3.73 & $(3.72 \pm 0.26 \pm 0.17) \times 10^{0}$ \\
\hline $4.00-5.00$ & 4.43 & $(2.89 \pm 0.16 \pm 0.16) \times 10^{0}$ & 4.43 & $(1.74 \pm 0.12 \pm 0.13) \times 10^{0}$ \\
\hline $5.00-6.00$ & 5.43 & $(1.35 \pm 0.14 \pm 0.08) \times 10^{0}$ & 5.43 & $(8.23 \pm 1.22 \pm 0.73) \times 10^{-1}$ \\
\hline $6.00-8.00$ & 6.80 & $(6.61 \pm 0.79 \pm 0.48) \times 10^{-1}$ & 6.83 & $(4.57 \pm 0.72 \pm 0.44) \times 10^{-1}$ \\
\hline $8.00-15.00$ & 10.11 & $(1.22 \pm 0.20 \pm 0.09) \times 10^{-1}$ & 10.25 & $(1.04 \pm 0.21 \pm 0.10) \times 10^{-1}$ \\
\hline $15.00-25.00$ & $18.16 \pm 0.03$ & $(3.43 \pm 2.46 \pm 0.27) \times 10^{-2}$ & $18.14 \pm 0.03$ & $(3.43 \pm 1.34 \pm 0.32) \times 10^{-2}$ \\
\hline
\end{tabular}


Table 5

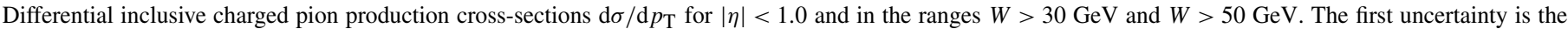
statistical uncertainty and the second uncertainty is the systematic uncertainty. No value is given if the error on $\left\langle p_{\mathrm{T}}\right\rangle$ is less than 0.01

\begin{tabular}{|c|c|c|c|c|}
\hline \multirow[b]{2}{*}{$p_{\mathrm{T}}[\mathrm{GeV}]$} & \multicolumn{2}{|l|}{$W>30 \mathrm{GeV}$} & \multicolumn{2}{|l|}{$W>50 \mathrm{GeV}$} \\
\hline & $\left\langle p_{\mathrm{T}}\right\rangle[\mathrm{GeV}]$ & $\mathrm{d} \sigma / \mathrm{d} p_{\mathrm{T}}[\mathrm{pb} / \mathrm{GeV}]$ & $\left\langle p_{\mathrm{T}}\right\rangle[\mathrm{GeV}]$ & $\mathrm{d} \sigma / \mathrm{d} p_{\mathrm{T}}[\mathrm{pb} / \mathrm{GeV}]$ \\
\hline $0.12-0.28$ & 0.20 & $(1.38 \pm 0.00 \pm 0.04) \times 10^{4}$ & 0.20 & $(6.71 \pm 0.02 \pm 0.22) \times 10^{3}$ \\
\hline $0.28-0.44$ & 0.35 & $(1.05 \pm 0.00 \pm 0.03) \times 10^{4}$ & 0.35 & $(5.11 \pm 0.01 \pm 0.17) \times 10^{3}$ \\
\hline $0.44-0.60$ & 0.51 & $(5.85 \pm 0.01 \pm 0.15) \times 10^{3}$ & 0.51 & $(2.85 \pm 0.01 \pm 0.09) \times 10^{3}$ \\
\hline $0.60-0.80$ & 0.69 & $(2.80 \pm 0.01 \pm 0.07) \times 10^{3}$ & 0.69 & $(1.37 \pm 0.01 \pm 0.04) \times 10^{3}$ \\
\hline $0.80-1.00$ & 0.89 & $(1.20 \pm 0.01 \pm 0.03) \times 10^{3}$ & 0.89 & $(6.04 \pm 0.04 \pm 0.19) \times 10^{2}$ \\
\hline $1.00-1.20$ & 1.09 & $(5.55 \pm 0.04 \pm 0.14) \times 10^{2}$ & 1.09 & $(2.83 \pm 0.03 \pm 0.08) \times 10^{2}$ \\
\hline $1.20-1.40$ & 1.29 & $(2.75 \pm 0.03 \pm 0.07) \times 10^{2}$ & 1.29 & $(1.43 \pm 0.02 \pm 0.04) \times 10^{2}$ \\
\hline $1.40-1.60$ & 1.49 & $(1.49 \pm 0.02 \pm 0.04) \times 10^{2}$ & 1.49 & $(7.88 \pm 0.14 \pm 0.20) \times 10^{1}$ \\
\hline $1.60-1.80$ & 1.69 & $(8.51 \pm 0.14 \pm 0.24) \times 10^{1}$ & 1.69 & $(4.58 \pm 0.10 \pm 0.12) \times 10^{1}$ \\
\hline $1.80-2.00$ & 1.89 & $(5.04 \pm 0.11 \pm 0.15) \times 10^{1}$ & 1.89 & $(2.77 \pm 0.08 \pm 0.08) \times 10^{1}$ \\
\hline $2.00-2.20$ & 2.09 & $(3.25 \pm 0.09 \pm 0.10) \times 10^{1}$ & 2.09 & $(1.80 \pm 0.06 \pm 0.06) \times 10^{1}$ \\
\hline $2.20-2.40$ & 2.29 & $(2.10 \pm 0.07 \pm 0.07) \times 10^{1}$ & 2.29 & $(1.15 \pm 0.05 \pm 0.04) \times 10^{1}$ \\
\hline $2.40-2.60$ & 2.50 & $(1.41 \pm 0.05 \pm 0.05) \times 10^{1}$ & 2.50 & $(7.90 \pm 0.41 \pm 0.33) \times 10^{0}$ \\
\hline $2.60-2.80$ & 2.70 & $(9.75 \pm 0.45 \pm 0.39) \times 10^{0}$ & 2.70 & $(5.43 \pm 0.33 \pm 0.23) \times 10^{0}$ \\
\hline $2.80-3.00$ & 2.90 & $(7.49 \pm 0.40 \pm 0.29) \times 10^{0}$ & 2.90 & $(4.19 \pm 0.30 \pm 0.18) \times 10^{0}$ \\
\hline $3.00-3.50$ & 3.23 & $(4.72 \pm 0.20 \pm 0.18) \times 10^{0}$ & 3.23 & $(2.79 \pm 0.16 \pm 0.11) \times 10^{0}$ \\
\hline $3.50-4.00$ & 3.73 & $(2.54 \pm 0.15 \pm 0.10) \times 10^{0}$ & 3.73 & $(1.53 \pm 0.12 \pm 0.07) \times 10^{0}$ \\
\hline $4.00-5.00$ & 4.43 & $(1.19 \pm 0.07 \pm 0.06) \times 10^{0}$ & 4.43 & $(7.03 \pm 0.56 \pm 0.51) \times 10^{-1}$ \\
\hline $5.00-6.00$ & 5.43 & $(5.99 \pm 0.68 \pm 0.38) \times 10^{-1}$ & 5.43 & $(3.66 \pm 0.58 \pm 0.32) \times 10^{-1}$ \\
\hline $6.00-8.00$ & 6.80 & $(2.85 \pm 0.36 \pm 0.21) \times 10^{-1}$ & 6.83 & $(1.99 \pm 0.33 \pm 0.19) \times 10^{-1}$ \\
\hline $8.00-15.00$ & 10.11 & $(5.27 \pm 0.93 \pm 0.40) \times 10^{-2}$ & 10.25 & $(4.28 \pm 0.91 \pm 0.40) \times 10^{-2}$ \\
\hline $15.00-25.00$ & $18.16 \pm 0.03$ & $(1.78 \pm 1.32 \pm 0.14) \times 10^{-2}$ & $18.14 \pm 0.03$ & $(1.80 \pm 0.78 \pm 0.17) \times 10^{-2}$ \\
\hline
\end{tabular}

transverse momenta than those measured by L3, leading to a disagreement between the two experiments at high transverse momenta and a better description of the OPAL data by NLO QCD than is the case for the L3 data.

\section{Acknowledgements}

We thank Bernd Kniehl for providing the NLO calculations and for many useful discussions. We particularly wish to thank the SL Division for the efficient operation of the LEP accelerator at all energies and for their close cooperation with our experimental group. In addition to the support staff at our own institutions we are pleased to acknowledge the Department of Energy, USA; National Science Foundation, USA; Particle Physics and Astronomy Research Council, UK; Natural Sciences and Engineering Research Council, Canada; Israel Science Foundation, administered by the Israel Academy of Science and Humanities; Benoziyo Center for High Energy Physics; Japanese Ministry of Education, Culture, Sports, Science and Technology (MEXT) and a grant under the MEXT International Science Research Program; Japanese Society for the Promotion of Science (JSPS); German Israeli Bi-national Science Foundation (GIF); Bundesministerium für Bildung und Forschung, Germany; National Research Council of Canada; Hungarian Foundation for Scientific Research, OTKA T-038240, and T-042864; The NWO/NATO Fund for Scientific Research, the Netherlands.

\section{References}

[1] L3 Collaboration, P. Achard, et al., Phys. Lett. B 554 (2003) 105.

[2] OPAL Collaboration, K. Ackerstaff, et al., Z. Phys. C 73 (1997) 433; OPAL Collaboration, G. Abbiendi, et al., Eur. Phys. J. C 10 (1999) 547; OPAL Collaboration, G. Abbiendi, et al., Eur. Phys. J. C 31 (2003) 307.

[3] L3 Collaboration, P. Achard, et al., Phys. Lett. B 602 (2004) 157.

[4] The OPAL Collaboration, K. Ackerstaff, et al., Eur. Phys. J. C 6 (1999) 253.

[5] OPAL Collaboration, K. Ahmet, et al., Nucl. Instrum. Methods A 305 (1991) 275 ;

S. Anderson, et al., Nucl. Instrum. Methods A 403 (1998) 326; OPAL Collaboration, G. Abbiendi, et al., Eur. Phys. J. C 14 (2000) 373.

[6] T. Sjöstrand, Comput. Phys. Commun. 82 (1994) 74.

[7] R. Engel, Z. Phys. C 66 (1995) 203; R. Engel, J. Ranft, Phys. Rev. D 54 (1996) 4244.

[8] G.A. Schuler, T. Sjöstrand, Z. Phys. C 68 (1995) 607.

[9] M. Glück, E. Reya, A. Vogt, Phys. Rev. D 45 (1992) 3986; M. Glück, E. Reya, A. Vogt, Phys. Rev. D 46 (1992) 1973.

[10] S. Jadach, B.F.L. Ward, Z. Was, Comput. Phys. Commun. 79 (1994) 503.

[11] J.A.M. Vermaseren, Nucl. Phys. B 229 (1983) 347.

[12] G. Marchesini, B.R. Webber, G. Abbiendi, I.G. Knowles, M.H. Seymour, L. Stanco, Comput. Phys. Commun. 67 (1992) 465.

[13] J. Allison, et al., Nucl. Instrum. Methods A 317 (1992) 47.

[14] OPAL Collaboration, G. Abbiendi, et al., Eur. Phys. J. C 14 (2000) 199.

[15] OPAL Collaboration, K. Ackerstaff, et al., Eur. Phys. J. C 2 (1998) 213; OPAL Collaboration, G. Abbiendi, et al., Eur. Phys. J. C 12 (2000) 567.

[16] J. Binnewies, B.A. Kniehl, G. Kramer, Phys. Rev. D 53 (1996) 6110; B.A. Kniehl, private communications.

[17] S. Albino, B.A. Kniehl, G. Kramer, Nucl. Phys. B 725 (2005) 181.

[18] P. Aurenche, J.-P. Guillet, M. Fontannaz, Z. Phys. C 64 (1994) 621. 